\title{
Prediction of Carotid Plaque Characteristics Using Non-Gated MR Imaging: Correlation with Endarterectomy Specimens
}

\author{
S. Narumi, M. Sasaki, H. Ohba, K. Ogasawara, M. Kobayashi, J. Hitomi, K. Mori, K. Ohura, M. Yamaguchi, K. Kudo, and Y. Terayama
}

\begin{abstract}
BACKGROUND AND PURPOSE: Electrocardiographic gating, commonly used in MR carotid plaque imaging, can negatively affect intraplaque contrast if the TR is inappropriate. The present study aimed to determine whether a non-gated technique with appropriate TRs can accurately evaluate intraplaque characteristics in specimens excised by CEA.
\end{abstract}

MATERIALS AND METHODS: We prospectively examined 40 consecutive patients who underwent CEA (59-82 years of age) by using a 1.5T scanner. Axial TIWI with a TR of $500 \mathrm{~ms}$ and PDWI and T2WI with a TR of $3000 \mathrm{~ms}$ with a self-navigated rotating-blade scan instead of cardiac gating were obtained. Signal intensities of the plaque and adjacent muscle were measured, and the CR on TIWI, PDWI, and T2WI as well as the gray-scale median on US were correlated with the pathologic findings of the CEA specimens.

RESULTS: On TIWI, the CRs of the carotid plaques differed significantly among groups in which the main components were histologically confirmed as fibrous tissue, lipid/necrosis, and hemorrhage (0.54-1.17, 1.16-1.53, and 1.40-2.29, respectively). The sensitivity and specificity for discriminating lipid/necrosis/hemorrhage from fibrous tissue were $96 \%$ and $100 \%$, respectively. On T2WI, the CRs of plaques with lipid/necrosis were significantly higher than those of other groups, but the CRs on PDWI and the gray-scale median on US were not significantly different among the groups.

CONCLUSIONS: Non-gated MR plaque imaging, particularly TIWI, can readily predict the intraplaque main components of the carotid artery with high sensitivity and specificity.

ABBREVIATIONS: CEA = carotid endarterectomy; $C R$ = contrast ratio; $E C G$ = electrocardiograph; MPRAGE = magnetization-prepared rapid acquisition of gradient echo; PDWI = proton-density-weighted imaging; US = ultrasonography

C arotid stenosis, either symptomatic or asymptomatic, can cause cerebral infarction and transient ischemic attack and is a possible indication for CEA or carotid artery stent placement. The severity of the stenosis is a strong risk factor for ischemic events and is widely used as a criterion for the selection of these therapies. ${ }^{1}$ In addition, components within atherosclerotic

Received October 5, 2011; accepted after revision March 18, 2012.

From the Department of Neurology and Gerontology (S.N., H.O., K.M., K.O., M.Y., Y.T.), Institute for Biomedical Sciences (M.S., K.K.), and Departments of Neurosurgery (K.O., M.K.) and Anatomy (J.H.), Iwate Medical University, Morioka, Japan.

This work was partly supported by a Research Grant for Cardiovascular Diseases (20C-1) from the Ministry of Health, Labor and Welfare of Japan and a Grant-in-Aid for Strategic Medical Science Research and Grants-in-Aid for Science Research (22890169, 22590963) from the Ministry of Education, Culture, Sports, Science and Technology of Japan.

Please address correspondence to Shinsuke Narumi, MD, Department of Neurology, Iwate Medical University, 19-one Uchimaru, Morioka 020-8505, Japan; e-mail: snarumi@iwate-med.ac.jp

- Indicates open access to non-subscribers at www.ajnr.org

http://dx.doi.org/10.3174/ajnr.A3249 plaques that cause stenoses are considered another important risk factor. Plaques consisting mainly of a lipid/necrotic core, intraplaque hemorrhage, or both with a thin fibrous cap, which are considered vulnerable plaques, are known to have a close correlation with ischemic events. ${ }^{2-4}$

The evaluation of intraplaque components for the determination of vulnerable plaques is commonly performed by using US. The echogenicity of the plaques, however, shows substantial overlap among components, even when using quantitative values such as the gray-scale median. ${ }^{5-7}$ In MR plaque imaging, which is also used to evaluate intraplaque components, a vulnerable plaque tends to show hyperintensity,,$^{2,3,8-15}$ but there are still substantial overlaps in signal intensity among different intraplaque components. ${ }^{2,8}$ Recent studies have suggested that this overlap can be attributed to the deterioration of image contrast due to the application of the ECG-gating technique as a motion-suppression method in MR plaque imaging, because this technique can cause the TR to become inappropriate and inconsistent. ${ }^{16,17} \mathrm{In}$ the present study, we attempted to determine whether non- 
gated MR plaque imaging with appropriate and fixed TRs can readily discriminate intraplaque components in patients who were candidates for CEA, by correlating the imaging results with the pathologic findings from CEA specimens.

\section{MATERIALS AND METHODS \\ Patients}

From April 2009 to July 2010, we prospectively examined 40 consecutive patients with stenosis of the cervical internal carotid artery who underwent CEA (40 men; age range, 59-82 years; mean age, 69.5 years). Of these patients, 30 had symptomatic stenosis of $\geq 70 \%$ and the others had asymptomatic stenosis of $\geq 80 \%$. The clinical characteristics of the patients included hypertension in 38 patients, hyperlipidemia in 22, and diabetes mellitus in 13 . All the examinations were performed after obtaining approval from the institutional review board and written informed consent from all patients.

\section{Imaging Protocol}

Axial T1WI, PDWI, and T2WI, respectively, of the affected carotid bifurcation were performed by using a $1.5 \mathrm{~T}$ MR imaging scanner (Echelon Vega; Hitachi Medical Corporation, Tokyo, Japan) and an 8-channel neurovascular coil. We used the following pulse sequence parameters: TR/TE, 500/12 ms (spin-echo T1WI) and 3000/12, $80 \mathrm{~ms}$ (dual-echo FSE PDWI/T2WI); FOV, $18 \mathrm{~cm}$; section thickness, $4.0 \mathrm{~mm}$ with intersection gaps of $1 \mathrm{~mm}$; number of sections, 9; and NEX, 2. A rotating-blade $k$-space acquisition technique with self-navigation, which is similar to the periodically rotated overlapping parallel lines with enhanced reconstruction method, ${ }^{18}$ was used for motion correction. In this technique, 40 radially rotating blades with 10 parallel phase-encoding lines and readout encoding of 256 steps, which caused oversampling at the center of the $k$-space, were obtained (acquisition time, 6 minutes 46 seconds [T1WI] and 8 minutes 4 seconds [PDWI/T2WI]), and mutual phase corrections among the blades were then performed as a self-navigation for compensating nonrigid motions. After rebinning to Cartesian coordinates with zero-fill interpolation, we obtained images with a matrix size of $512 \times 512$ (apparent pixel size, $0.35 \times 0.35 \mathrm{~mm}$ ). Chemical shift selective saturation pulses within the planes and nonselective saturation pulses at the superior and inferior sides were used as fat-suppression and black-blood imaging methods, respectively. The section direction was carefully set in a plane perpendicular to the long axis of the carotid bifurcation on sagittal 2D phase-contrast MR angiographic images. 3D B-mode and color Doppler images of the affected carotid bifurcations were also obtained in all patients by using a 3D US scanner (Voluson 730 Expert; GE Healthcare, Milwaukee, Wisconsin) and a 5- to 12-MHz probe.

\section{Histologic Evaluation}

Specimens excised in an en bloc fashion from the affected carotid arteries were submitted for histologic evaluation. After they were fixed in formaldehyde, transverse sections of the internal carotid arteries were obtained with careful setting of the direction and position corresponding to those of the MR images. During the histologic preparation, hematoxylin-eosin, Masson trichrome, and anti-Glycophorin-A stains were applied to paraffin-embedded 7 - $\mu \mathrm{m}$-thick sections. An independent researcher, who was unaware of the clinical and imaging findings, measured areas of fibrous, lipid/necrotic, and hemorrhagic components with a manual tracing method by using a software package (ImageJ, Version 1.44; National Institutes of Health, Bethesda, Maryland). The principal intraplaque components, in which the area of one of the components was $\geq 50 \%$ of the total plaque area and was $10 \%$ larger than the other components, were then determined.

\section{Data Processing and Statistical Analyses}

For quantitative evaluation of MR images, the signal intensity of the carotid plaque and the adjacent sternomastoid muscle was measured on the image with the maximum plaque size. The plaques and muscles were manually traced 3 times in a blind and random fashion by using the polygon cursor of a free software package (ExaVision Lite; Ziosoft, Tokyo, Japan), and the signal intensity values obtained were averaged. The $\mathrm{CR}$ was calculated by dividing the signal intensity of the plaque by that of the muscle.

For quantitative analysis of US images, axial images corresponding to the MR and sagittal images from 3D datasets were generated and exported in a JPEG format. The gray-scale median values of the plaques were then measured in a blind fashion by manual tracing with a polygon cursor after normalization of the gray-scale (0 for the arterial lumen and 195 for the arterial adventitia) by using a graphic software package (Photoshop CS4; Adobe Systems, San Jose, California), as reported previously. ${ }^{7}$

Differences in the CR on T1WI, PDWI, and T2WI as well as the gray-scale median on US in plaques containing different components were examined by using the Kruskal-Wallis test followed by a post hoc Mann-Whitney $U$ test. To determine the sensitivity and specificity of the images for the prediction of main intraplaque components, we also performed receiver operating characteristic analyses. Cutoff values were determined by using the Youden index. The $\alpha$ level used was .05.

\section{RESULTS}

MR plaque imaging and 3D-US imaging of the carotid bifurcation, in addition to CEA, were performed on all patients. However, the quality of the PDWI and T2WI in 1 patient and the 3D US image in 3 patients was suboptimal due to strong motion artifacts and severe calcification, respectively. Black- blood effects of the carotid arteries on the MR images were sufficient in all patients. Owing to the results of the histologic measurements, 10 patients were excluded because no intraplaque component fulfilled the criteria for determining the principal component. Therefore, the remaining 30 patients (59-79 years; mean age, 68.9 years) were eligible for further quantitative analyses. Measurements of intraplaque components on histologic specimens showed that the main component of plaques was fibrous tissue in 8 cases (64\%-92\% occupancy), lipid/necrosis in 12 cases $(50 \%-$ $94 \%$ occupancy), and hemorrhage in 10 cases (50\%-70\% occupancy).

For the T1WI, the CRs of the carotid plaques containing mainly fibrous tissue, lipid/necrosis, and hemorrhage were in the range of $0.54-1.17$ (median, 0.97), 1.16-1.53 (median, 1.27), and $1.40-2.29$ (median, 1.99), respectively, and were significantly dif- 
ferent among the 3 groups $(P<.001$, Kruskal-Wallis and Mann-Whitney $U$ tests) (Table and Figs 1-5). For the T2WI, the CRs of the plaques were in the range of 1.51-3.79 (median, 1.89), 2.34-5.10 (median, 2.88), and 1.47-3.10 (median, 1.89), respectively, and significant differences were observed only between lipid/ necrosis and hemorrhage $(P=.041$, Kruskal-Wallis test; $P=.025$, MannWhitney $U$ test) (Table and Figs 1-5). In contrast, for the PDWI, the CRs of the plaques were in the range of 0.84-1.52 (median, 1.33), 0.86-1.82 (median, 1.43), and 1.08-1.82 (median, 1.53), respectively, and no significant differences were observed $(P=$ .07 , Kruskal-Wallis test), though the values showed tendencies similar to those measured on the T1WI (Table and Figs 1-5). The gray-scale medians of the carotid plaques on the US images did not show significant differences among the groups: 4-18 (median, 7.5), 1-25 (median, 13), and 1-29 (median, 16), respectively ( $P=$ .26 , Kruskal-Wallis test) (Table and Figs 1-5).

Receiver operating characteristic analyses showed that for the T1WI, the sensitivities and specificities for discriminating plaques with hemorrhage or lipid/necrosis from those with fibrous tissue were $96 \%$ and $100 \%$, respectively, when the cutoff value of the CR was set at 1.17. Those for discriminating hemorrhage from others were $90 \%$ and $100 \%$, respectively, with a cutoff value of 1.55. For T2WI, the sensitivities and specificities for discriminating lipid/necrosis from other components were $100 \%$ and $65 \%$, respectively, at a cutoff value of 2.30 , and $92 \%$ and $100 \%$, respectively, when combined with cutoff values of $>1.17$ and $<1.55$ for T1WI (Figs 1 and 2).

\section{DISCUSSION}

It is crucial that plaque imaging techniques achieve sufficient contrast to discriminate lipid, necrosis, and hemorrhage from fibrous tissue, to identify vulnerable plaque consisting mainly of a lipid/ necrotic core and/or intraplaque hemorrhage with a thin fibrous cap. In the present study, the main components of the carotid plaque were distinguished with high sensitivity and

${ }^{a} P<.001$.
Contrast ratios of the carotid plaques to adjacent muscle on MR plaque imaging and US

\begin{tabular}{lcccc}
\hline & \multicolumn{3}{c}{ Main Plaque Component } & \\
\cline { 2 - 4 } Imaging & $\begin{array}{c}\text { Fibrous Tissue } \\
(\boldsymbol{n}=8)\end{array}$ & $\begin{array}{c}\text { Lipid/Necrosis } \\
(\boldsymbol{n}=12)\end{array}$ & $\begin{array}{c}\text { Hemorrhage } \\
(\boldsymbol{n}=10)\end{array}$ & $\begin{array}{c}\text { P Value } \\
\text { (Kruskal-Wallis Test) }\end{array}$ \\
\hline MR & & $1.16-1.53(1.27)^{\mathrm{a}}$ & $1.40-2.29(1.99)^{\mathrm{a}}$ & $<.001$ \\
TIWI & $0.54-1.17(0.97)^{\mathrm{a}}$ & $0.86-1.82(1.43)$ & $1.08-1.82(1.53)$ & .070 \\
PDWI & $0.84-1.52(1.33)$ & $2.34-5.10(2.88)^{\mathrm{b}}$ & $1.47-3.10(1.89)$ & .041 \\
T2WI & $1.51-3.79(1.89)$ & $1-25(13)$ & $1-29(16)$ & .26 \\
US & $4-18(7.5)$ & 110 & \\
GSM & & &
\end{tabular}

Note:-GSM indicates gray-scale median.

${ }^{\mathrm{b}} P<.05$, Mann-Whitney $U$ test; data are presented as range (median).
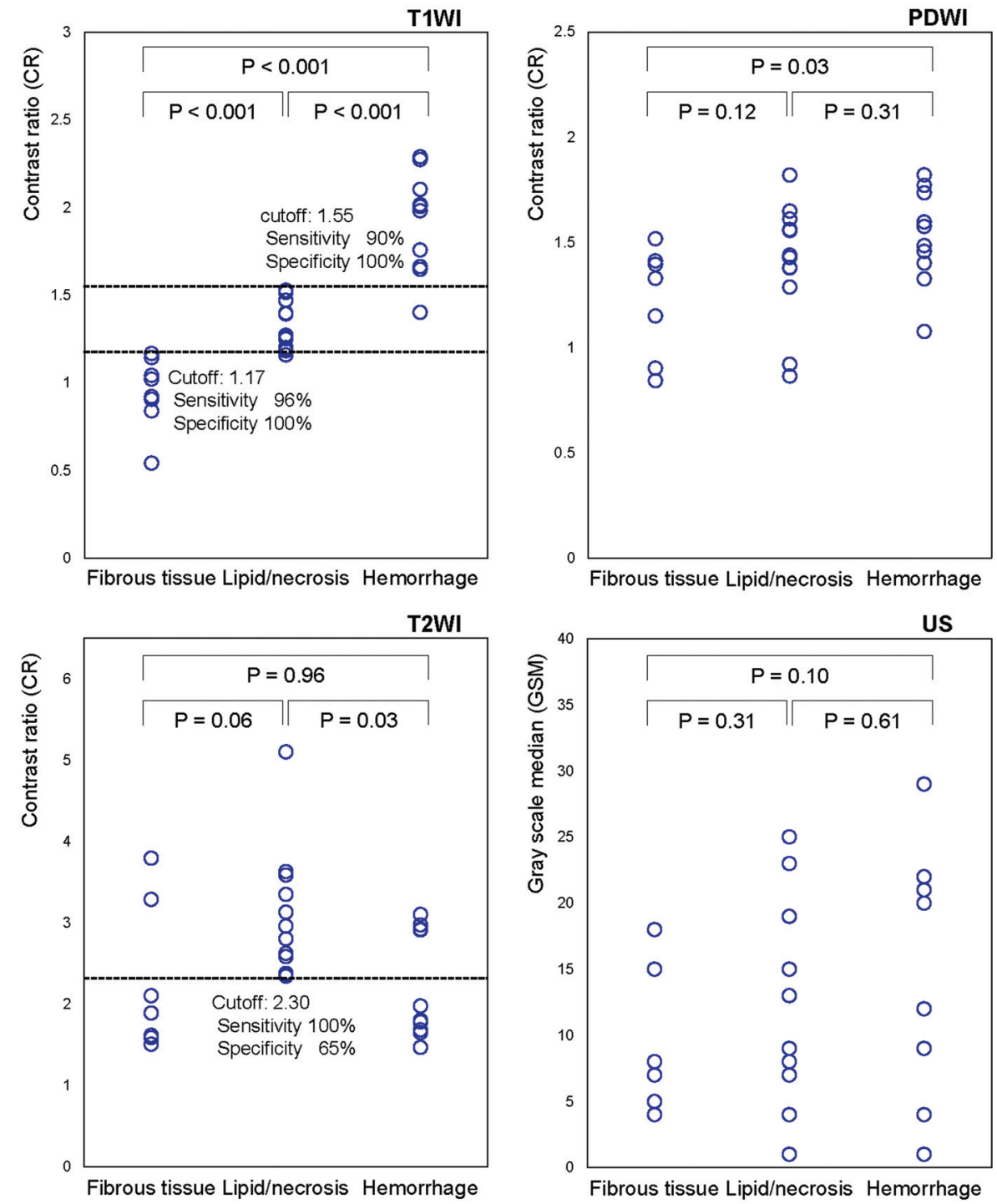

FIG 1. Correlation between non-gated MR plaque imaging or US and histologic analysis of carotid plaques. On TIWI, the CRs of the carotid plaques to the adjacent muscle are significantly different between plaques containing mainly fibrous tissue, lipid/necrosis, and hemorrhage. On T2WI, plaques containing lipid/necrosis show significant hyperintensity. In contrast, no differences are noted in PDWI or gray-scale median on US.

specificity by using a nongated method, suggesting that the method introduced in this study can readily identify vulnerable plaque.

The sensitivity and specificity of T1WI for detecting intra- 


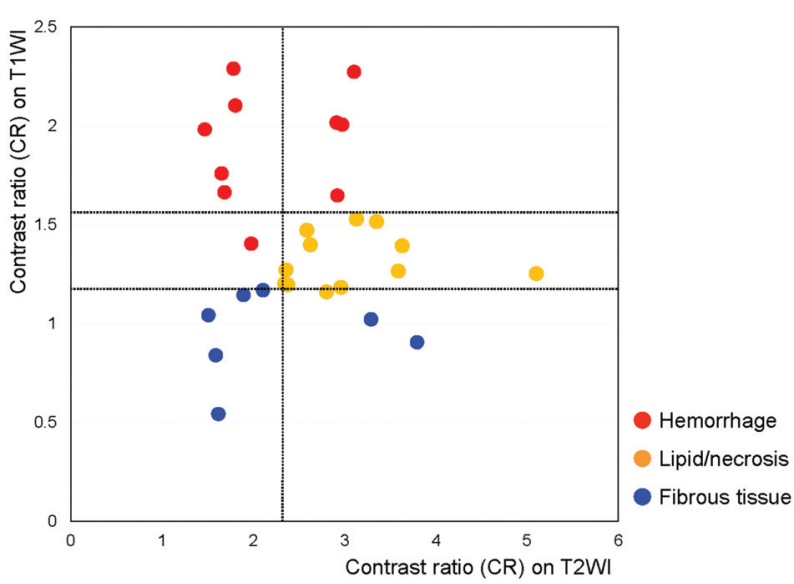

FIG 2. Distribution of the CRs of the carotid plaque on non-gated TIWI and T2WI. Intraplaque characteristics can be determined with minimal overlaps by the combined use of imaging techniques. Blue, orange, and red circles show the plaques containing mainly fibrous tissue, lipid/necrosis, and hemorrhage, respectively.

plaque hemorrhage were $90 \%$ and $100 \%$, respectively, which were higher than the values that have been reported by using different T1WI techniques (70\%-84\% and $84 \%-97 \%$, respectively). ${ }^{19,20}$ These were also comparable with the sensitivity and specificity achieved by using combined imaging techniques (85\%-95\% and $70 \%-92 \%$, respectively). ${ }^{13,21}$ In the discrimination of plaques consisting of lipid/necrosis/hemorrhage from those consisting of fibrous tissue, the sensitivity and specificity of T1WI in this study were $96 \%$ and $100 \%$, respectively, which were significantly higher than the T1WI values previously reported (79\% and $84 \%$, respec- tively $)^{2}$ and were comparable with those of combined imaging methods (93\%-96\% and 93\%-96\%, respectively). ${ }^{8,15}$

The excellent contrast of the images obtained in the present study can be attributed to the rejection of the ECG-gating method for motion correction and selection of appropriate TRs. In the ECG-gating method, the TR of T1WI is forced to be $1 \mathrm{R}-\mathrm{R}$ interval, resulting in TRs of $800-1000 \mathrm{~ms}$, which is too long to maintain T1-related contrast. ${ }^{16,17}$ Similarly, the TRs of ECG-gated PDWI and T2WI with 2 R-R intervals are usually 1400-2000 ms, which is too short to preserve the contrast. Hence, we adopted fixed TRs of 500 and $3000 \mathrm{~ms}$ for T1WI and PDWI/T2WI, respectively, which are commonly used in routine clinical imaging. The present results show that CRs on non-gated T1WI exhibited small overlaps among plaques with different components, and nongated PDWI showed significant overlaps, suggesting that T1WI should be used instead of PDWI to discriminate plaque components, which can be achieved only by the nongated method.

Although previous studies reported the use of non-gated spin-echo methods for T1WI, the relatively long TRs used, such as $800 \mathrm{~ms}$, can reduce T1-weighting and can negatively affect image contrast. ${ }^{13,16,21}$ Other studies adopted a modified MPRAGE technique to enhance T1-related contrast. ${ }^{9,19}$ In this technique, however, setting of the TI for suppressing the blood signal can attenuate the signal of the lipid component that mainly consists of cholesterols. ${ }^{17}$ In contrast, the chemical shift selective saturation pulses we used can maintain the signal of the cholesterol-rich lipid component while selectively suppressing the signal of the subcutaneous fat. The present T1WI measurements provided more accurate results than those ob-
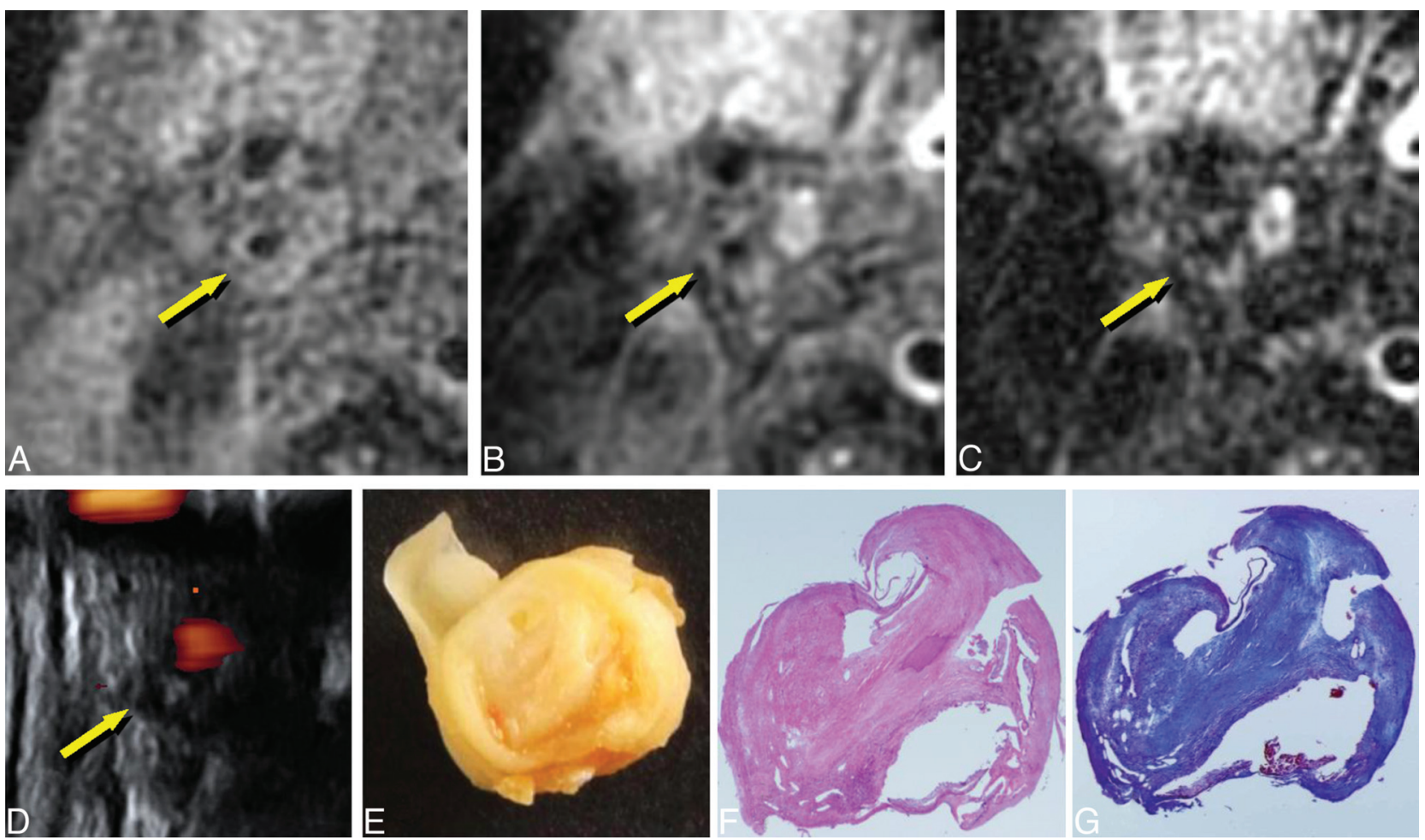

FIG 3. Imaging of carotid plaque containing mainly fibrous tissue (75-year-old man with right carotid stenosis). A, TIWI. B, PDWI. C, T2WI. D, US. $E$, macroscopic specimen. F, Histologic specimen with hematoxylin-eosin staining. G, Histologic specimen with Masson trichrome staining. The plaque shows isointensity to adjacent muscle ( $A-C$, arrows) and low echoic areas ( $D$, arrow). Staining of the specimens shows that the plaque consists of thick fibrous tissue ( $E-G, 4 \times$ magnification). 

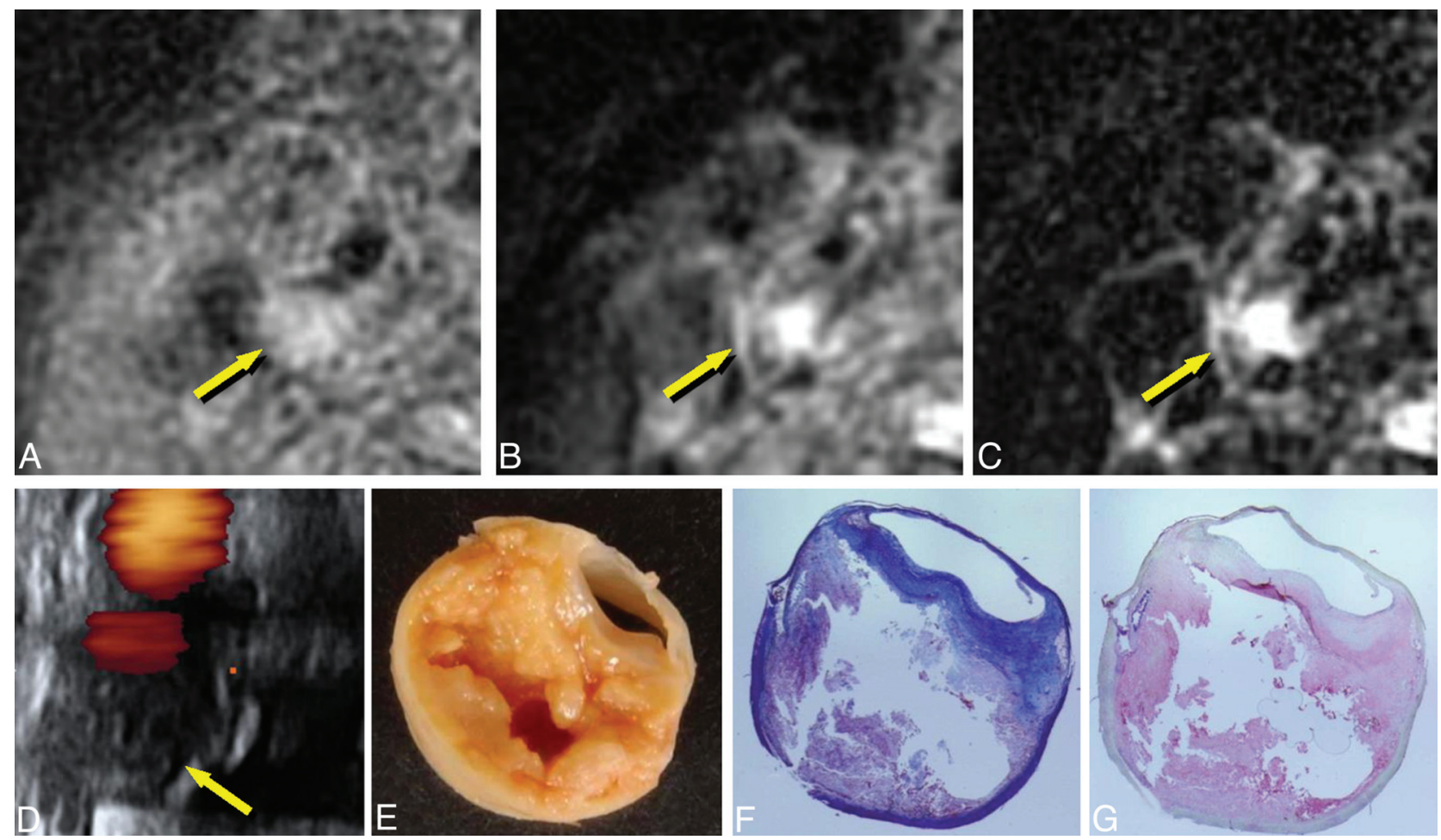

FIG 4. Imaging of carotid plaque containing mainly lipid and/or necrosis (72-year-old man with right carotid stenosis). A-E, Same as in Fig 3. $F$, Masson trichrome staining. G, Anti-glycophorin-A staining. The plaque shows mild hyperintensity on TIWI and PDWI ( $A$ and $B$, arrows) and distinct hyperintensity on $\mathrm{T} 2 \mathrm{WI}(C$, arrow); low echoic areas are seen on US ( $D$, arrow). The plaque contains necrotic tissue and lipid, as seen on the specimens ( $E-G, 4 \times$ magnification).
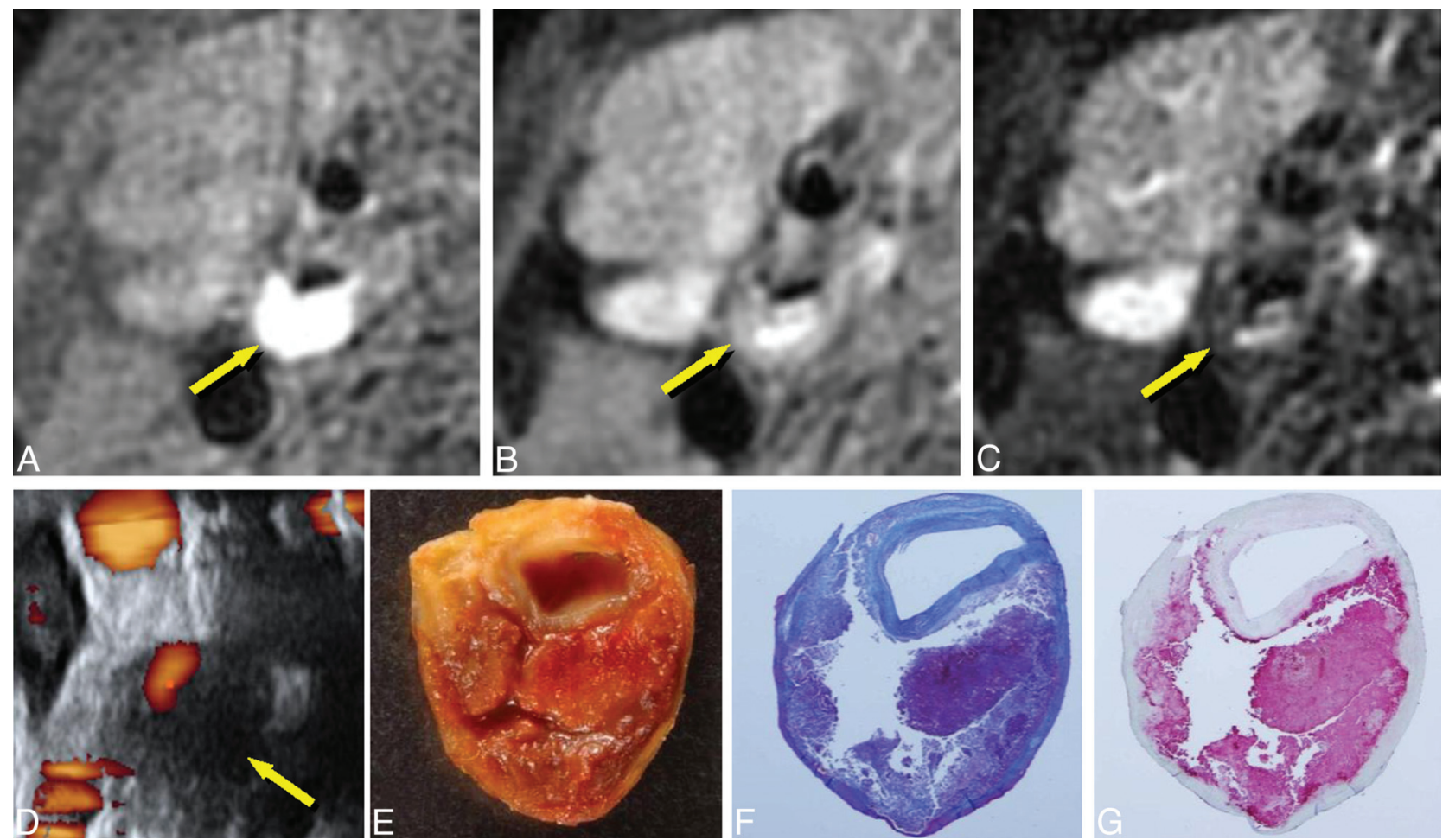

FIG 5. Imaging of carotid plaque containing mainly hemorrhage (61-year-old man with right carotid stenosis). A-G, Same as in Fig 4 . The plaque shows evident hyperintensity on TIWI ( $A$, arrow), moderate hyperintensity on PDWI and T2WI ( $B$ and $C$, arrows), and low echoic areas on US ( $D$, arrow). The plaque contains massive hemorrhage ( $E-G, 4 \times$ magnification).

tained in prior studies, ${ }^{13,19-21}$ indicating the potential superiority of non-gated spin-echo T1WI, which is supported by the recent report regarding direct comparisons between non-gated spin-echo and MPRAGE methods. ${ }^{17}$ 
In addition to T1WI, we found that the CRs of lipid-/necrosisrich plaques tended to show hyperintensity on non-gated T2WI, presumably owing to a sufficiently long TR and TE. These results suggest that non-gated T2WI can be useful for the discrimination of lipid/necrosis from other components. The combined use of non-gated T1WI and T2WI with the appropriate scanning parameters can therefore accurately estimate intraplaque components, as suggested by previous reports. ${ }^{8,13,15,21}$ Furthermore, this method is simple and easy to use and will enable the achievement of quantitative assessments as image contrast becomes constant among patients who have different heart rates.

In the present study, the self-navigated rotating-blade technique was used instead of the ECG-gating method for suppressing motion artifacts caused by pulsation of cervical arteries. This technique enables the correction of misregistrations related to rigid and nonrigid motions, which is based on oversampling at the center of the $k$-space and self-navigation, such as mutual phase, rotation, and translation corrections among rotating trajectories. ${ }^{18}$ In addition, the technique yields an increase in the number of sections because standard presaturation pulses can be used as a black-blood method, while the multiple inversion recovery method, characterized by marked restriction on the multisection acquisition, is essential as a black-blood method for ECG-gated imaging. ${ }^{22}$ In this technique, classic presaturation pulses can sufficiently suppress flow signal within the stenosis because the data are obtained regardless of the cardiac phase, whereas the multiple inversion recovery method is essential in the ECG-gating technique because data are obtained only during the diastolic phase, in which flow velocity is minimal. The self-navigated rotating-blade technique is therefore a more suitable method than ECG-gating for obtaining high-contrast multisection images while keeping minimal motion artifacts and sufficient black-blood effects.

We compared non-gated MR plaque imaging with US imaging, which is widely used for characterizing intraplaque components. On US, hypoechoic plaque is generally considered to reflect a vulnerable plaque, though this finding is subjective. Hence, we measured the gray-scale median of the plaques on sections corresponding to MR images for quantitative and direct comparison. However, the gray-scale median values showed a marked overlap among groups with different intraplaque components and showed no significant differences. This result suggests that nongated MR plaque imaging is more accurate than US for the identification of intraplaque components.

The present study has several limitations. First, the signal intensity of the entire plaque measured on MR plaque imaging was compared with the main component within the plaque on histologic specimens. Because carotid plaques can contain heterogeneous components, our results may include errors due to partial volume effects among the different components. In addition, we did not assess calcific components within the plaques because patients with poor findings on US images due to strong calcifications were excluded from further analyses. Hence, small inconspicuous calcification would likely affect the quantitative values to some extent. Further investigations by using pixel-by-pixel analyses of $3 \mathrm{D}$ data with multimodal coregistration techniques are needed, though these are beyond the scope of this study, to overcome these issues,. Nevertheless, non-gated T1WI, followed by
T2WI, can readily determine the main intraplaque components. These images are therefore thought to possess sufficient contrast for regional characterization within the plaque with a pixel-bypixel method applying appropriate cutoff values, which is currently under investigation.

A second limitation is that we did not perform a direct comparison between the non-gated spin-echo method and other methods such as ECG-gated FSE, MPRAGE, 3D time-of-flight MR angiography, and 3D-FSE, which have been used as T1WI in previous studies. ${ }^{9,19,23}$ It was thought that additional scans to achieve these images would need excessive acquisition times, which would be unacceptable to the patients. However, a previous study reported that relatively long TR settings, which are comparable with TRs under ECG-gating, can deteriorate the contrast between stable and unstable components on T1WI, suggesting a potential disadvantage of the ECG-gated method. ${ }^{16}$ In addition, another recent study determined the discrepancies among 4 different methods of T1WI by direct comparison and revealed the advantages of non-gated spin-echo T1WI over other techniques. ${ }^{17}$ We also decided against performing contrast-enhanced T1WI because it is expensive, time-consuming, and relatively invasive. Contrast-enhanced T1WI is used to evaluate the thickness of the fibrous cap because an enhancement effect can occur only in fibrous tissue through the vasa vasorum. Further studies are needed to determine whether nongated nonenhanced images can identify the fibrous cap as effectively as contrast-enhanced T1WI.

Another limitation of this study is that instead of the pair of surface coils frequently used in previous studies, we used a standard neurovascular coil that is more applicable to clinical situations. To achieve a relatively high signal-to-noise ratio, we had to compromise on spatial resolution. However, we attained signal homogeneity within sections, which can improve the precision of quantitative assessment by using the CRs to the adjacent muscle. Selection of a coil system during MR plaque imaging is another issue that requires further investigation.

Generally, MR plaque imaging remains complicated and timeconsuming, and the scanning techniques, parameter settings, coil systems, and evaluation methods show marked variation among institutions, which should be minimized. This study reveals that the simple technique of non-gated T1WI can accurately discriminate among the main components of carotid plaques, indicating that this method is effective for standardizing and simplifying plaque imaging. Further prospective multicenter/multivendor studies are needed to validate this hypothesis.

\section{CONCLUSIONS}

Non-gated T1-weighted MR plaque imaging could readily characterize the main intraplaque components in patients who underwent CEA, with small overlaps and high sensitivity and specificity.

Disclosures: Makoto Sasaki-UNRELATED: Consultancy: Lundbek,* Mitsubishi Pharma, ${ }^{*}$ Actelion, ${ }^{*}$ Grants/Grants Pending: Japanese Ministries, ${ }^{*}$ Travel/Accommodations/Meeting Expenses Unrelated to Activities Listed: GE Healthcare, Daiichi Pharma, Takeda Pharma, Bayer, OTHER: Makato Sasaki is a consultant for Hitachi Medical Corporation and has received honoraria from Hitachi Medical Corporation and GE Healthcare. Jiro Hitomi-RELATED: Grant: Adaptable and Seamless Technology Transfer Program through target-driven research and development, Japan Science and Technology Agency, ${ }^{*}$ Support for Travel to Meetings for the Study or Other Purposes: Adaptable and Seamless Technology Transfer Program through 
target-driven research and development, Japan Science and Technology Agency, ${ }^{*}$ UNRELATED: Board Membership: Japan Society for the Promotion of Science, Committee for Grants-in-Aid for Scientific Research, Grants/Grants Pending: Adaptable and Seamless Technology Transfer Program through target-driven research and development, Japan Science and Technology Agency, ${ }^{\star}$ Royalties: Igaku-Shoin Ltd (Tokyo), Maruzen (Tokyo), Comments: textbooks. Kohsuke Kudo-UNRELATED: Consultancy: Hitachi Medical Systems, ${ }^{\star}$ Payment for Lectures (including service on Speakers Bureaus): GE Healthcare, Hitachi Medical Systems.* Yasuo TerayamaUNRELATED: Consultancy: Otsuka Pharmaceuticals, Sanofi-Aventis, Glaxo-SmithKline, Grants/Grants Pending: Ministry of Health, Labor, and Welfare, Payment for Lectures (including service on Speakers Bureaus): Otsuka Pharmaceuticals, SanofiAventis, Glaxo-Smith-Kline, Novartis, Pfizer. *Money paid to the institution.

\section{REFERENCES}

1. North American Symptomatic Carotid Endarterectomy Trial Collaborators. Beneficial effect of carotid endarterectomy in symptomatic patients with high-grade carotid stenosis. N Engl J Med 1991; 325:445-53

2. Yoshida K, Narumi O, Chin M, et al. Characterization of carotid atherosclerosis and detection of soft plaque with use of black-blood MR imaging. AJNR Am J Neuroradiol 2008;29:868-74

3. Takaya N, Yuan C, Chu B, et al. Association between carotid plaque characteristics and subsequent ischemic cerebrovascular events: a prospective assessment with MRI-initial results. Stroke 2006; 37:818-23

4. Altaf N, MacSweeney ST, Gladman J, et al. Carotid intraplaque hemorrhage predicts recurrent symptoms in patients with high-grade carotid stenosis. Stroke 2007;38:1633-35

5. Waki H, Masuyama T, Mori H, et al. Ultrasonic tissue characterization of the atherosclerotic carotid artery: histological correlates or carotid integrated backscatter. Circ J 2003;67:1013-16

6. Sabetai MM, Tegos TJ, Nicolaides AN, et al. Reproducibility of computer-quantified carotid plaque echogenicity: can we overcome the subjectivity? Stroke 2000;31:2189-96

7. Sztajzel R, Momjian S, Momjian-Mayor I, et al. Stratified gray-scale median analysis and color mapping of the carotid plaque: correlation with endarterectomy specimen histology of 28 patients. Stroke 2005;36:741-45

8. Watanabe Y, Nagayama M, Suga T, et al. Characterization of atherosclerotic plaque of carotid arteries with histopathological correlation: vascular wall MR imaging vs. color Doppler ultrasonography (US). J Magn Reson Imaging 2008;28:478-85

9. Yamada N, Higashi M, Otsubo R, et al. Association between signal hyperintensity on T1-weighted MR imaging of carotid plaques and ipsilateral ischemic events. AJNR Am J Neuroradiol 2007;28:287-92

10. Zhao X, Miller ZE, Yuan C. Atherosclerotic plaque imaging by carotid MRI. Curr Cardiol Rep 2009;11:70-77
11. Yuan C, Mitsumori LM, Beach KW, et al. Carotid atherosclerotic plaque: noninvasive MR characterization and identification of vulnerable lesions. Radiology 2001;221:285-99

12. Fabiano S, Mancino S, Stefanini M, et al. High-resolution multicontrast-weighted MR imaging from human carotid endarterectomy specimens to assess carotid plaque components. Eur Radiol 2008;18:2912-21

13. Chu B, Kampschulte A, Ferguson MS, et al. Hemorrhage in the atherosclerotic carotid plaque: a high-resolution MRI study. Stroke 2004;35:1079-84

14. Saam T, Ferguson MS, Yarnykh VL, et al. Quantitative evaluation of carotid plaque composition by in vivo MRI. Arterioscler Thromb Vasc Biol 2005;25:234-39

15. Cappendijk VC, Cleutjens KB, Kessels AG, et al. Assessment of human atherosclerotic carotid plaque components with multisequence MR imaging: initial experience. Radiology 2005;234: 487-92

16. Narumi S, Sasaki M, Ohba H, et al. Altered carotid plaque signal among different repetition times on T1-weighted magnetic resonance plaque imaging with self-navigated radial-scan technique. Neuroradiology 2010;52:285-90

17. Saito A, Sasaki M, Ogasawara K, et al. Carotid plaque signal differences among four kinds of T1-weighted magnetic resonance imaging techniques: a histopathological correlation study. Neuroradiology 2012 Mar 17. [Epub ahead of print]

18. Pipe JG. Motion correction with PROPELLER MRI: application to head motion and free-breathing cardiac imaging. Magn Reson Med 1999;42:963-69

19. Moody AR, Murphy RE, Morgan PS, et al. Characterization of complicated carotid plaque with magnetic resonance direct thrombus imaging in patients with cerebral ischemia. Circulation 2003;107:3047-52

20. Ota H, Yarnykh VL, Ferguson MS, et al. Carotid intraplaque hemorrhage imaging at 3.0-T MR imaging: comparison of the diagnostic performance of three T1-weighted sequences. Radiology 2010; 254:551-63

21. Yuan C, Mitsumori LM, Ferguson MS, et al. In vivo accuracy of multispectral magnetic resonance imaging for identifying lipid-rich necrotic cores and intraplaque hemorrhage in advanced human carotid plaques. Circulation 2001;104:2051-56

22. Song HK, Wright AC, Wolf RL, et al. Multislice double inversion pulse sequence for efficient black-blood MRI. Magn Reson Med 2002;47:616-20

23. Balu N, Chu B, Hatsukami TS, et al. Comparison between 2D and 3D high-resolution black-blood techniques for carotid artery wall imaging in clinically significant atherosclerosis. J Magn Reson Imaging 2008;27:918-24 